\title{
Two-dimensional Protein Electrophoresis: From Molecular Pathway Discovery to Biomarker Discovery in Neurological Disorders
}

\author{
Leila H. Choe, Brenda G. Werner, and Kelvin H. Lee \\ School of Chemical and Biomolecular Engineering, Cornell University, Ithaca, New York 14853
}

\begin{abstract}
Summary: Two-dimensional protein electrophoresis (2-DE) has undergone many technical improvements in the past 30 years, resulting in an analytical method that is unparalleled in the resolution of complex protein mixtures and capable of quantifying changes in protein expression from a wide variety of tissues and samples. The technique has been applied in many studies of neurologic disease to identify changes in spot patterns that correlate with disease. The true power of the technique emerges when it is coupled to state-of-the-art methods in mass spectrometry, which enable identification of the protein or proteins contained within a spot of interest on a 2-DE map. Investigators have successfully applied the technique to gain
\end{abstract}

improved understanding of neurologic disease mechanisms in humans and in animal models and to discover biomarkers that are useful in the clinical setting. An important extension to these efforts that has not been realized thus far is the desire to profile changes in protein expression that result from therapy to help relate disease-modifying effects at the molecular level with clinical outcomes. Here we review the major advances in 2-DE methods and discuss specific examples of its application in the study of neurologic diseases. Key Words: Proteomics, two-dimensional electrophoresis, neurological disorders, Alzheimer's disease, Creutzfeldt-Jakob disease, biomarkers.

\section{INTRODUCTION}

Proteomics refers to the study of the comprehensive representation of the proteins expressed by a genome at a given time. While the genome of an organism is static, its corresponding proteome is a responsive and dynamic entity that widely differs within the organism. It can respond to and reflect environmental influences, developmental stages, and states of health and disease. Stateof-the-art proteomics technologies have been applied to a wide variety of studies for the separation, visualization, and analysis of a heterogeneous and abundant array of proteins from complex biofluids, tissues, and cells. Proteomic studies commonly employ two-dimensional electrophoresis (2-DE), a technique that has evolved over 30 years to the current state of technology and implementation. Two-dimensional electrophoresis permits highresolution separation of individual proteins from complex mixtures, in two orthogonal dimensions. While each

Address correspondence and reprint requests to: Kelvin H. Lee, $\mathrm{Ph} . \mathrm{D}$., School of Chemical and Biomolecular Engineering, 120 Olin Hall, Cornell University, Ithaca, NY 14853-5201. E-mail: KHL9@ cornell.edu dimension alone has the capability to resolve up to an average of 200 different protein species, the sequential use of both significantly expands this capacity, with the potential to resolve as many as 10,000 proteins within one sample, ${ }^{1}$ although in practice detection of $2000-$ 5000 proteins is more common. ${ }^{2}$ This resolving power is significant, as a eukaryotic cell proteome may consist of an array of at minimum 10,000 different proteins, exhibiting a broad range of protein concentrations, for example, from $10^{1}$ to $10^{6}$ copies per cell. ${ }^{2}$

Despite some limitations, the overall advantages and relative accessibility of 2-DE technology has made it an attractive methodology in the study of neurological diseases. Earlier works resulted in some qualitative and quantitative discoveries, but the lack of protein characterization limited their impact. Recent advancements in technology have renewed interest in 2-DE methodology and its application in this field. Biomarker discovery and disease mechanism studies can benefit from the fact that 2-DE enables the simultaneous survey of the protein content of a given sample, including the detection of and distinction between different modifications of a particular protein, such as post-translational modifications and 
cleavage products. An added advantage, in cases in which sample availability is limited, is that these gelseparated proteins also can be physically archived in the gel matrix after image acquisition. This allows for protein characterization at a later date without the need to repeat the experiment.

Current applications attempt to address multiple aspects of neurologic diseases. Definitive and differential antemortem diagnoses of some neurologic diseases, such as Alzheimer's disease (AD), remain a critical challenge. The precise cause and mechanism of disease pathology, such as improper beta amyloid $(\mathrm{A} \beta)$ cleavage and the formation of neurofibrillary tangles, are still largely unidentified. As a result of these challenges associated with diagnosis and poorly understood mechanism, effective therapeutics and treatment targets for many neurodegenerative diseases have not been developed. Published efforts to address these issues intersect and overlap, some with consistent results, others with conflicting results. To date, an enormous amount of proteomics data has been generated (with and without 2-DE technology) on biological fluids and tissues, protein isoforms and interactions, and primary and secondary pathways, providing a growing basis of information that can be used to further propel and guide proteomic discoveries in neurologic diseases.

In this review we present first a description of general practices and major advances in 2-DE methodology with no emphasis on neurologic disorders. We then discuss considerations and current applications of 2-DE to neurological disorders.

\section{TWO-DIMENSIONAL ELECTROPHORESIS: MAJOR TECHNOLOGICAL ADVANCES}

\section{Background}

Essential work performed in 1975 combined the previously established methodologies of isoelectric focusing (IEF) and polyacrylamide gel electrophoresis (PAGE) in a two-dimensional format, effectively separating sample proteins in the first dimension by isoelectric point and in the second dimension according to molecular mass. ${ }^{3,4}$ Two-dimensional separations enabled the analysis and resolution of complex and dynamic protein mixtures found in biofluids, tissues, and cells, producing a visual protein map unique to a particular sample at a particular time. The technology was soon applied to analysis of plant and mammalian tissue proteins. ${ }^{5,6}$ Klose was among the first to use protein mapping and analysis in biomarker discovery and used a mouse model. ${ }^{4}$ Early implementations of 2-DE were challenged by a lack of reproducibility and tools to easily identify and measure proteins within a sample. Today, many improvements have been made in the methodology, yet 2-DE still faces challenges in interlaboratory reproducibility and sample preparation, ${ }^{7}$ especially with regard to low abundance proteins and hydrophobic proteins.

\section{Sample preparation}

Sample preparation is an important first step in 2-DE analysis, which serves multiple functions. It must ensure solubilization of proteins, prevent aggregation and hydrophobic interactions, and remove interfering components such as nucleotides and excess salts, while maximizing protein recovery and minimizing artifactual or chemical alterations. Ultimately, sample preparation will determine which proteins are capable of being resolved on the resultant 2D gel. However, proteins exist within a broad spectrum of physicochemical properties and abundance levels. Because of a wide range of isoelectric points, molecular weights, and hydrophobicity represented within a given sample, a single survey of all proteins from a complex biological sample is currently unachievable with existing technology.

A sample preparation protocol must be optimized for a particular type of sample. For example, a protocol for cerebrospinal fluid (CSF), which does not normally contain a significant number of cells, cannot effectively be applied to brain tissue, which contains substantial cell content and is high in lipid content. In general, most protocols involve an initial treatment such as microdialysis, precipitation, or sonication to remove common contaminants such as nucleic acids, salt ions, and lipids, followed by the addition of denaturants, reducing and alkylating agents, detergents, and chaotropes to facilitate protein solubility. ${ }^{7,8}$ Reductants such as dithiothreitol (DTT) and dithioerythritol (DTE) are commonly used to prevent protein oxidation and reduce disulfide bonds. Resulting sulfhydryl groups are often alkylated with iodoacetamide or iodoacetic acid to prevent reformation of intra- and interprotein disulfide bonds. A chaotrope, commonly urea or in combination with thiourea, is used to disrupt noncovalent interactions, resulting in the elimination of secondary and tertiary protein structure, solubilization of some hydrophobic proteins, and reduction of protein-protein interactions. The choice of detergents is usually limited to nonionic (Triton X-100, Nonidet NP-40) or zwitterionic detergents [3-(3-cholamidopropy1)dimethylammonio-1-propane sulfonate (CHAPS)], to avoid charge interference during isoelectric focusing; these are often used to disrupt hydrophobic interactions and increase solubility. Protease inhibitors may also be added to minimize proteolytic degradation.

Recently, the use of sample prefractionation before or after sample preparation has emerged in proteomic studies. Because high abundance proteins may comprise upwards of $90 \%$ of the total protein expressed by a cell, ${ }^{1}$ sample "complexity reduction" has been actively studied. A few examples of complexity reduction are subcellular fractionation ${ }^{9}$ to isolate certain organelles of inter- 
est, liquid $\mathrm{IEF}^{10}$ to increase resolution in a given $\mathrm{pH}$ range, or affinity chromatography ${ }^{11}$ to remove high abundance proteins. Complexity reduction has been applied in plasma, serum, and CSF studies to remove high abundance proteins such as albumin and immunoglobins with the intention of unmasking and enriching lower abundance proteins. However, it has been shown that such an approach may have undesired results in the removal of other proteins that may interact with or bind to the high abundance proteins as well as removal of some low abundance proteins. ${ }^{12}$ While the goal of this type of prefractionation is to enrich proteins of interest, in practice, it can result in selective, unintentional losses. Creative separation and prefractionation strategies are sometimes required to isolate proteins with relatively extreme properties; however, in general, a reduction in the nature and number of procedural manipulations will improve reproducibility and minimize protein loss.

\section{The first dimension: isoelectric focusing}

Early implementations of 2-DE used in the first dimension hand-generated IEF $\mathrm{pH}$ gradients created with mixtures of acidic and basic low-molecular-weight carrier ampholytes in a gel. Upon application of an electric field, carrier ampholytes migrate toward their respective isoelectric point where they become uncharged; direction and speed of migration is influenced by their charge. A significant problem with carrier ampholyte-based IEF is gradient destabilization, caused by long migration times, the unfixed nature of carrier ampholytes in free solution, and subsequent cathodic drift. Furthermore, as the process of creating carrier ampholyte gradients is involved and lengthy, significant batch-to-batch and technical variations in the $\mathrm{pH}$ gradient formed increased variability in IEF results.

A significant improvement in IEF was achieved with the development of immobilized $\mathrm{pH}$ gradients (IPG) by Bjellqvist. ${ }^{13}$ Immobilized gradients are covalently created, using a mixture of immobilines or acrylamide derivative buffers copolymerized in a gradient with the gel matrix. A fixed $\mathrm{pH}$ gradient is created, thus eliminating problems of $\mathrm{pH}$ or cathodic drift or distortions typically resulting from carrier ampholyte gradients and buffers; reproducibility of resultant spot patterns is additionally improved. $^{14}$

Prefabricated, quality controlled, commercially available strips offer reproducibility and reliability, enabling improved intra- and interlaboratory comparisons of data, and are commonly used today. These IPG strips are dehydrated upon fabrication and enable sample application via in-gel rehydration, combining IPG strip rehydration and sample application in one step. ${ }^{15}$ Alternatively, samples may be cup-loaded onto rehydrated IPG strips; cup-loading has been described as a strategy to improve loading efficiency of high-molecular-weight and hydro- phobic proteins. ${ }^{2}$ Narrow $\mathrm{pH}$ range IPG strips (1 pH unit) have been reported to result in threefold improvements in protein spot resolution and detection of low-abundance proteins. ${ }^{16}$ Although this strategy has its benefits in resolution, its application to discovery-based global protein profiling is more limited because it requires additional IEF runs.

\section{The second dimension: polyacrylamide gel electrophoresis}

After IEF, the IPG strips typically undergo an equilibration step in which focused proteins are resolubilized, coated with sodium dodecyl sulfate (SDS), and disulfide bonds are reduced and alkylated. The SDS binds to proteins in proportion to the protein mass, overriding intrinsic charges and allowing separation in the second dimension based on protein mass alone. SDS-PAGE is a robust and well-established technique ${ }^{17}$ used for the orthogonal separation by protein mass.

Current implementations apply IEF-separated samples to the second dimension by placing IPG strips in direct contact with the slab gel border, allowing protein migration from one gel to another. A variety of gel compositions and formats, including a range of gel sizes, pore sizes, gradients, and mechanical strengths, are available to accommodate broad and specific protein profiles. Tools and equipment to run mini-format 2-DE gels are widely available and have the benefit of a much shorter turnaround time at the expense of decreased resolution. Larger format 2-DE gels provide more resolution for the same amount of sample ${ }^{18}$ but are also more difficult to physically manipulate.

\section{Protein visualization and quantitation}

Protein visualization and detection tools should be sensitive enough for detection of low-abundance proteins as well as exhibit capability in a wide dynamic and linear range. Detection procedures should also be reproducible and compatible with downstream protein characterization, particularly mass spectrometry. Total protein stains such as colloidal Coomassie blue, silver stain, and SYPRO Ruby are among the most sensitive nonradiolabeling methods used today for 2D gels. Silver staining, performed as early as 1979 by Switzer, ${ }^{19}$ exhibits high sensitivity, $0.1 \mathrm{ng}$, with a 10 - to 40 -fold linear range in 2D spots. ${ }^{2}$ While highly sensitive, silver staining methods are lengthy and complex, and the stain is generally incompatible with mass spectrometry. Colloidal Coomassie blue ${ }^{20}$ is a background-free stain that can detect as little as $15 \mathrm{ng}$ per 2-DE spot. ${ }^{21}$ This stain is generally compatible with mass spectrometry and its use is generally less complex than silver staining. Fluorescent staining with SYPRO Ruby is a recent alternative that is relatively simple to perform, fast, and sensitive. It has a linear dynamic range of $1-1000 \mathrm{ng}$ and is generally compatible with protein identification methods. ${ }^{22}$ The 
limit of detection of SYPRO Ruby has been reported by others to be as low as $0.5 \mathrm{ng}^{23}$

There is a wide range of alternative and complementary detection methods that can enable direct quantitation, multiplexing of samples, or selective detection. Some methods require introduction of the label before 2-DE separation. Radiolabeled amino acids can be substituted into culture media where they are incorporated into newly synthesized proteins in vivo. ${ }^{24}$ These metabolically labeled proteins are run on 2-DE and imaged on film or by storage phosphor technology. Difference gel electrophoresis (DIGE) labels proteins during sample preparation and also offers the advantage of a multiplex experiment in one gel. ${ }^{25}$ DIGE employs three spectrally distinct cyanine dyes of matching charge and molecular weight to label up to three different samples. Labeled samples are mixed and run as a single sample on one gel. Subsequently, the gel is scanned at a different wavelength corresponding to each of the dyes, resulting in three superimposable images. The intent is to minimize gel-to-gel variability and improve relative quantitation among samples.

Other detection methods are applied postseparation. If an antibody against a protein of interest exists, 2-DE immunoblots can detect multiple forms and fragments of a protein on a gel. ${ }^{26}$ Specific post-translational modifications can be probed over an entire gel using stains such as ProQ Diamond for phosphorylated proteins ${ }^{27}$ and ProQ Emerald for glycosylated proteins, ${ }^{28}$ either stain can be used in series with a total protein stain. Protein oxidation and nitration are also detectable using an immunoblot technique. ${ }^{29,30}$

Gel scanners are critical tools for the capture of 2-DE gel images. The sensitivity and dynamic range of the gel scanner should match or exceed that of the stain employed to preserve data integrity. Currently, laser fluorescence-based scanners are capable of capturing a dynamic range of five orders of magnitude and producing 16-bit images with $25 \mu \mathrm{m}$ resolution. Image analysis software is also important in maintaining the integrity of the protein profile. Computer-assisted analysis is widely used as it offers automation in detection, matching, and relative quantitation of spots among different gels. Some current software packages, for example, ImageMaster (GE Healthcare, Piscataway, NJ) and Progenesis (Nonlinear Dynamics, Durham, NC), offer improved spot distinction, better spot matching among nonalike spot patterns, and statistical tools for data analysis.

\section{Protein identification and characterization}

The full potential of 2-DE analyses is realized only when coupled to protein mass spectrometry and other protein characterization methods. Previously, N-terminal amino acid sequencing ${ }^{31}$ or antibody immunoblots were the only methods available to identify the proteins in spots of interest. Although these methods are still used today, mass spectrometry has made protein identification more accessible and in some cases more informative because of the ability to assign a gene or protein name to a spot and/or to characterize post-translational modifications.

Accordingly, 2-DE serves two key purposes. First, the 2D separation provides the means to analyze the samples for spots of interest, and second, it serves as a micropreparative protein purification method for downstream characterization. Spots of interest are excised and typically undergo in-gel digestion by a sequence-specific protease. The resultant peptides are then analyzed by mass spectrometry. Several types of mass spectrometers are available for protein analysis; these technologies are described in more detail elsewhere in this issue $\mathrm{e}^{32}$ as well as by Finehout and Lee. ${ }^{33}$ In general, one type of analysis generates a peptide mass fingerprint for each spot based on the measurement of the mass-to-charge ratio of the proteolyzed peptides. By comparing observed masses to an in silico digest of all available protein sequences, a high probability protein assignment can be made. An important extension is the ability to perform tandem mass spectrometry. Here, a specified peptide is subjected to further analysis in the mass spectrometer and an amino acid sequence can be inferred from the resulting spectrum. This workflow has enabled accurate identification of proteins in the femtomole range. ${ }^{34}$ It is important to highlight that the protein identifications are based on probabilities and that subsequent validation of the protein assignment remains a critical and often neglected step in proteomics studies.

\section{Summary}

Considerable improvements in sensitivity, resolution, and reproducibility in 2-DE methodology, as well as advances in mass spectrometry and bioinformatics tools have established 2-DE as a core proteomics discovery tool enabling accurate and reliable detection of alterations in protein expression and metabolic modifications in carefully designed experiments. Protein isolation kits, sample preparation kits, IPG strips, polyacrylamide slab gels, protein stains, and the equipment to run, visualize, and analyze 2-DE gels are commercially available. As a result, intra- and interlaboratory 2-DE reproducibility has improved. Nonetheless, 2-DE is still a technique that involves many manual manipulations and attention must be given to performing these steps consistently from experiment to experiment to ensure reproducibility because many of these steps cannot be easily automated. Choe and Lee ${ }^{35}$ studied the impact of sample preparation variability and found a $3-14 \%$ coefficient of variation $(\% \mathrm{CV})$ for qualitative changes among replicate gels. They also report an increase in \%CV for qualitative changes as an effect of automation-dependent image 
analysis. Nishihara and Champion ${ }^{36}$ found a $3-33 \% \mathrm{CV}$ for quantitative variation among replicate gels using multiple image analysis software packages. Molloy and coworkers ${ }^{37}$ attributed an average of $23 \% \mathrm{CV}$ in technical variation to the 2-DE process itself. They also report on the variability found in 2-DE experiments based on different types of samples.

There are several recent reviews for further discussion on recent advances, applications, and limitations in 2-DE proteomic methodologies. ${ }^{38-40}$

\section{APPLICATIONS IN NEUROLOGICAL DISORDER RESEARCH}

Neurologic disease etiology and development is often a result of multigenic features influenced by environmental factors and cellular events. Disease incidence due to monogenic causality has been estimated to be only $2 \% .{ }^{41}$ Given this complexity, protein expression data have become a valuable resource to aid in understanding the molecular pathology of neurologic disease. Early efforts combining 2-DE and protein mass spectrometry were concentrated on the global detection and identification of proteins from a given sample. Two-dimensional electrophoresis protein maps served an important role, as a catalog of both protein localization and sample specificity and as a benchmark for understanding diverse disease states. Comprehensive 2-DE protein maps have been published for several samples related to neurology, including human CSF, ${ }^{42}$ plasma, ${ }^{43}$ and brain. ${ }^{44}$ Today, the diversity and specialization of sample types, the rapid pace of data generation, and the accessibility to technology for identifying spots from 2-DE studies have diminished the role and applicability of publicly available protein maps. The current emphasis in 2-DE based research is on relating specific changes between protein profiles to the underlying biology.

There have been a large number of studies that apply 2-DE to the study of neurologic disease for the discovery of biomarkers and in the understanding of mechanism in a general sense. Because of the immense general literature, we have chosen to focus this section only on studies that rely on 2-DE to understand disease mechanism, biomarker discovery, and disease diagnosis and only discuss results that offer a definitive protein identification. For simplicity, we consider mechanistic studies as those involving brain tissue and we categorize biomarker studies as those involving CSF or plasma. There is a variety of literature that is not covered but the reader is referred to an excellent review by Fountoulakis. ${ }^{45}$

\section{Molecular mechanisms}

Studies of molecular pathways in neurologic disease are typically conducted using brain tissue because the brain is the primary site of neuropathological change. A common study design for brain tissue involves a comparison between normal controls and disease samples with the goal of identifying differentially or uniquely expressed proteins. Ideally, changes in protein expression will correlate to some element of the disease, its progression, or possible therapeutic targets. A recent review summarizes a large volume of 2-DE comparisonbased findings in brain tissues from the following: $\mathrm{AD}$, Down's syndrome, Parkinson's disease, schizophrenia, bipolar disorder, and depression. ${ }^{46}$ An extension to the efforts covered in that review is the use of 2-DE to study protein post-translational modifications related to disease mechanism. Proteolytic activity and post-translational modifications have been suspected to play various roles in neurodegenerative diseases. For example, aberrant glycosylation and hyperphosphorylation of tau have been reported to play a role in neurodegenerative tauopathies as reviewed in Gong and coworkers, ${ }^{47}$ and abnormal metabolism of amyloid precursor protein has been linked to $\mathrm{AD}$ as reviewed in Golde and coworkers. ${ }^{48}$ Kanninen and coworkers ${ }^{49}$ used a glycoprotein stain on 2-DE gels to detect and compare glycosylated proteins in the frontal cortex of AD patients and controls. They detected 50 glycosylated proteins out of 150 total proteins and found differential glycosylation levels for collapsin response mediator protein 2 (decreased by $79 \%$ in $\mathrm{AD}$ ) and an unidentified protein (increased by $1200 \%$ in AD).

Oxidative stress is a particularly active area of research in this field and is discussed in greater detail elsewhere in this issue. ${ }^{50}$ Oxidative stress and its resultant protein, lipid, and DNA modifications are believed to play a critical role in the onset or development of age-related disorders and disease-mediated neurodegeneration. ${ }^{51}$ Using a 2-DE carbonyl immunoblot for oxidation detection, Aksenov and coworkers ${ }^{29}$ found increased levels of oxidation in regions of the brain that had pathophysiological changes due to AD compared with the same regions in control and in a relatively spared region of AD brain. Castegna and coworkers ${ }^{52}$ employed a 2-DE carbonyl immunoblot for the detection of protein oxidation in the inferior parietal lobe of AD patients and controls. They found statistically significant increases in the oxidation levels of creatine kinase BB, ubiquitin carboxy-terminal hydrolase L-1, and glutamine synthetase. Castegna and coworkers $^{30}$ later applied a similar technique to find nitration targets in $\mathrm{AD}$ brain samples. Using a 2-DE immunoblot with an antibody against 3-nitrotyrosine, they detected increased immunoreactivity in alpha enolase, triosephosphate isomerase, and neuropolypeptide $\mathrm{h} 3$ in AD brain samples compared with controls.

Two-dimensional electrophoresis can also been used in protein interaction studies. In a pilot proteomic study, Cottrell and coworkers ${ }^{53}$ immunoprecipitated amyloid precursor protein (APP) and its bound protein complexes from the hippocampi and cortical tissues of $\mathrm{AD}$ patients 
and controls. These complexes were separated using 2-DE and are reported to contain proteins from the following functional classes: molecular chaperones, cytoskeletal and structural proteins, proteins involved in trafficking, adaptors, and enzymes. Twenty-one proteins were identified from the complexes in both normal and AD samples, of which Fe65, 14-3-3, $\alpha$ B-crystallin, dynein, and dynamin-1 were confirmed by Western blot or immunohistochemistry. This study relied on a mouse model of AD shown to express moderate levels of human APP for immunohistochemical confirmations.

Animal models attempt to generate specific neuropathologies under tightly controlled conditions that reduce genetic or environmental effects that are inherent to human studies and seek to closely mimic at least one critical aspect of disease pathology and neurochemistry in the disorder of interest. The difficulty in obtaining human brain tissue in general, particularly at different stages of disease, has led to the use of animal models to supplement studies in human neurological disorders. Although studies of animal models cannot provide exact insights into human pathology, they do offer an important estimate of relevant changes. Tilleman and coworkers ${ }^{54} \mathrm{em}-$ ployed transgenic mice (htau40-1) and wild-type mice to study the proteomic effects of overexpression of the longest isoform of human tau (tau40). Two-dimensional electrophoresis and MS revealed 13 proteins that were significantly ( $p \leq 0.05$ ) increased in htau40-1 samples and 5 that were decreased. These 18 proteins were grouped into the following categories: chaperones, redoxproteins, components of the pyruvate dehydrogenase complex, blood-related proteins, cytoskeleton-associated proteins, proteins involved in energy metabolism, glycolysis and signal transduction, and other proteins.

David and coworkers ${ }^{55}$ studied brain tissue from transgenic mice $(\mathrm{P} 301 \mathrm{~L})$ that express the human pathogenic mutation P301L of tau and human tau40 and wild-type mice to identify 30 proteins that were significantly $(p<$ 0.05 ) altered in expression by 2-DE gels. Based on the functional categories of these proteins (metabolism and mitochondrial respiration, oxidative stress, and synapse function), they conducted analyses for related function and activity. They report that 2-DE and functional analysis suggest mitochondrial dysfunction as a result of tau accumulation in the absence of neurofibrillary tangles.

\section{Biomarkers and disease diagnosis}

Discoveries in brain tissue can provide insight into the molecular pathology of a disease and also lead to potential biomarkers for disease presence, stage, or response to therapy. However, routine clinical use of any potential protein biomarker is most effective when applied to samples that are relatively easy to obtain. This situation poses a challenge in biomarker discovery. Biomarkers for neurologic disease may not be easily identified in plasma because of the relatively high concentration of protein and the presence of the blood-brain barrier that may hinder the discovery of any biochemical changes from the central nervous system (CNS). Ventricular CSF is straightforward to obtain on autopsy when a definite diagnosis of neurodegenerative disease is generally available; whereas antemortem lumbar CSF, coupled to a definite diagnosis on postmortem exam, is more difficult to obtain for some diseases unless a lumbar puncture is indicated to establish a clinical diagnosis for the suspected disease. Unfortunately, studies of antemortem CSF from patients with definitive diagnosis, preferably from large cohorts, are the only studies that can improve upon the current state of the art in clinical diagnosis. Studies that rely on antemortem CSF with no definite diagnosis will have limited impact. While we cannot provide an exhaustive review of all CSF-based 2-DE studies, we can highlight some important examples and discuss relevant considerations.

Antemortem CSF protein expression is representative of brain biochemistry and blood-brain barrier function and may reflect both normal and pathological end products. While changes that result from the molecular pathology of the disease in brain can lead to biomarkers that can be identified in CSF, changes in CSF protein expression may also result indirectly from the disease process and may be useful as biomarkers. Such surrogate biomarkers, if statistically validated, can be equally important endpoints in biomarker discovery.

One important example of the use of 2-DE to identify biomarkers of disease involves Creutzfeldt-Jakob disease (CJD). Two-dimensional gel analysis of $541 \mathrm{CSF}$ samples revealed two unique spots that consistently appeared in CJD samples compared with normal and neurologic controls. ${ }^{56}$ These spots were subsequently identified as 14-3-3 proteins. ${ }^{57}$ The discovery of these 14-3-3 proteins led to the development of an immunoassay used for presumptive clinical diagnosis, a simpler, less expensive, and more rapid test format than 2-DE. While this immunoassay exhibits $96 \%$ sensitivity and $96 \%$ specificity for sporadic CJD, ${ }^{57}$ the test is not as sensitive for variant CJD where it was found that only $50 \%$ of confirmed variant CJD cases exhibit positive results for 14$3-3 .^{58}$ Today, the 14-3-3 biomarker is widely used for the diagnosis of sporadic CJD ${ }^{59}$ and the World Health Organization has revised their clinical criteria for this diagnosis to include a positive 14-3-3 test. ${ }^{60}$ This result highlights one important feature of the application of 2-DE in biomarker studies: 2-DE functions best as a discovery tool and the final clinical implementations of these discoveries are likely to rely on more traditional methods such as immunoassays. Although the 14-3-3 marker may not be as sensitive in the differential diagnosis of sporadic and variant CJD, other potential biomarkers have shown promise in this regard; Choe and 
coworkers ${ }^{61}$ used 2-DE to identify one particular isoform of apolipoprotein $\mathrm{E}$ that has potential to differentiate sporadic and variant CJD.

One of the most actively studied diseases is AD, which has several CSF protein biomarkers that demonstrate some ability to differentiate AD subjects from normal and demented control subjects. The best known CSF AD biomarkers are $\mathrm{A} \beta$, total tau, and phosphorylated tau, which were initially studied as biomarkers based on an understanding of disease pathology and not as a result of 2-DE analysis. Two-dimensional electrophoresis analysis of CSF has been used to help identify alternate and complementary markers. Davidsson and coworkers ${ }^{62}$ employed 2-DE, SYPRO Ruby staining, and mass spectrometry to analyze antemortem CSF for alterations in protein expression. Six proteins and their isoforms, primarily apolipoproteins, were found to have differential expressions between AD (not including definitive diagnoses) and normal controls; the authors suggested that differences in apolipoprotein levels may indicate an altered cholesterol metabolism in the AD brain. This group later related these findings to results from a study of frontotemporal dementia (FTD) CSF and CSF from normal controls. ${ }^{63}$ Here, apolipoprotein expression was less affected than in the AD study. They also identified six proteins with differential expression between FTD and controls. One protein in particular, granin-like neuroendocrine precursor (ProSAAS), had not previously been reported in CSF. The authors report that the differential protein expressions in one disease versus the other may be due to differences in disease pathology.

A biomarker does not need to be unique to one disease. Differential expression levels of proteins common to both diseased and healthy samples or common among diseased samples have potential to be used in diagnosis. Finehout et al. ${ }^{64}$ used 2-DE and mass spectrometry to study AD, Parkinson's disease, multiple sclerosis, and neurosyphilis CSF. Isoforms of several complement proteins, C $3 \mathrm{~b}, \mathrm{C} 4 \mathrm{~b}$, and complement factors $\mathrm{B}$ and $\mathrm{H}$, exhibited a significant $(p<0.05)$ change. In addition, the differential expression of these isoforms was found to be statistically significant between most pair-wise comparisons of diseases.

While premortem CSF has been the basis for the majority of biomarker studies, postmortem CSF has been examined as a suggested model of massive brain insult and for the detection of biomarkers for ischemia and neurodegeneration. ${ }^{26}$ Analysis of postmortem CSF revealed 13 protein spots that were significantly increased in postmortem CSF from one set of subjects compared with antemortem CSF (control) from a different set of subjects. Identification and further analysis of these proteins led to the discovery of prostaglandin D2 synthase (PGDS) isoforms specific to control samples. A 2D immunoblot found acidic isoforms, thought to be linked to
$\mathrm{N}$-glycosylation, only in the control sample. In a follow-up study, this group validated their prostaglandin D2 synthase findings using 50 antemortem CSF samples representing various neurological disorders and healthy controls. ${ }^{65}$ After confirming the presence and relative locations of the isoforms from a $2 \mathrm{D}$ immunoblot to an IEF immunoblot, the remainder of the study was conducted in IEF immunoblots. Analysis of the normalized volume ratio of acidic to basic isoforms revealed a significant decrease $(p<0.001)$ in the AD group compared with healthy controls, as well as a significant difference $(p<0.05)$ between the $\mathrm{AD}$ and amyotrophic lateral sclerosis groups.

As mentioned above, surrogate biomarkers may not be a direct result of the molecular pathology of disease but may be an indirect result of the presence of disease. Indeed, it has been suggested that some neurological disorders may actually be of a systemic nature with manifestations throughout the body. ${ }^{66}$ Thus, biomarker discovery for neurologic diseases need not be restricted to the tissues of the CNS and can be reasonably extended to include plasma, which is more convenient to obtain. Choi et al. ${ }^{67}$ employed 2-DE analysis and carbonyl immunoblot chemistry to discover significant changes in the oxidation of isoforms of fibrinogen $\gamma$-chain precursor protein and $\alpha$-1-antitrypsin precursor in AD plasma compared with control plasma. Changes in oxidation of isoforms of human transferrin, hemopexin, and $\alpha$-1-antitrypsin were detected in prefractionated plasma from $\mathrm{AD}$ compared with control patient plasma samples, suggesting new potential biomarkers of AD.$^{68}$ Others have studied protein expression changes in red blood cells and lymphocytes in AD patients versus healthy controls. ${ }^{69,70}$

We have presented a survey of some of the directions and challenges of current biomarker research supporting neurological disorder diagnosis. A recent review provides a summary of some of this work employing antemortem $\mathrm{CSF}^{71}$ and comprehensive reviews of biomarker discovery as applied to specific neurological disorders have also been recently published for $\mathrm{AD}^{72}$ and CJD. ${ }^{59}$ For one viewpoint on the direction of biomarker discovery in disease diagnosis, the reader is directed to another recent review ${ }^{73}$ that presents additional discussion regarding issues in biomarker discovery and diagnosis, including experimental design and validation, statistical analysis, and selection of control and disease groups.

\section{CONCLUSIONS}

Two-dimensional electrophoresis has undergone many technical improvements since the original report, resulting in an analytical method that is unparalleled in the resolution of complex protein mixtures and capable of quantifying changes in protein expression from a wide variety of tissues and samples. The technique has already 
been applied in numerous studies of neurologic disease to identify changes in spot patterns that correlate with disease. However, the true power of the technique emerges when it is coupled to state-of-the-art methods in mass spectrometry, which makes it possible to identify the protein or proteins that correspond to an observed relevant change in spot intensity on a 2-DE map. In this mode, investigators have successfully applied the technique to gain an improved understanding of disease mechanisms in humans and in animal models and to discover biomarkers that are useful in the clinical setting. An important extension to these efforts that has not been realized to date is the desire to profile changes in protein expression that result from therapy to better relate disease-modifying effects at the molecular level with clinical outcomes.

The complexity and multigenic nature that underlies most neurologic diseases reinforces the importance of developing multivariate diagnostic methods. Two-dimensional electrophoresis is a technique that generates multivariate data based on the simultaneous profiling of protein expression. Ultimately, however, the discoveries made using 2-DE must be validated with other techniques and in prospective studies. The lack of validation and further study has limited the number of effective biomarker studies based on 2-DE and other proteomics methods in this emerging field, but the pipeline of 2-DE discoveries offers tremendous promise.

Acknowledgments: This work was supported in part by the National Institutes of Health (MH59926), the New York State Office of Science, Technology, and Academic Research, and the Institute for the Study of Aging, Cornell University.

\section{REFERENCES}

1. Gabor Miklos GL, Maleszka R. Protein functions and biological contexts. Proteomics 1:169-178, 2001.

2. Görg A, Weiss W, Dunn MJ. Current two-dimensional electrophoresis technology for proteomics. Proteomics 4:3665-3685, 2004.

3. O'Farrell PH. High resolution two-dimensional electrophoresis of proteins. J Biol Chem 250:4007-4021, 1975.

4. Klose J. Protein mapping by combined isoelectric focusing and electrophoresis of mouse tissues: a novel approach to testing for induced point mutations in mammals. Humangenetik 26:231-243, 1975.

5. Iborra F, Buhler JM. Protein subunit mapping: a sensitive high resolution method. Anal Biochem 74:503-511, 1976.

6. Scheele GA. Two-dimensional gel analysis of soluble proteins: characterization of guinea pig exocrine pancreatic proteins. J Biol Chem 250:5375-5385, 1975.

7. Shaw MM, Riederer BM. Sample preparation for two-dimensional gel electrophoresis. Proteomics 3:1408-1417, 2003.

8. Rabilloud T. Solubilization of proteins for electrophoretic analysis. Electrophoresis 17:813-829, 1996.

9. Huber LA, Pfaller K, Vietor I. Organelle proteomics: implications for subcellular fractionation in proteomics. Circ Res 92:962-968, 2003.

10. Zuo X, Speicher DW. A method for global analysis of complex proteomes using sample prefractionation by solution isoelectrofocusing prior to two-dimensional electrophoresis. Anal Biochem 284:266-278, 2000.
11. Björhall K, Miliotis T, Davidsson P. Comparison of different depletion strategies for improved resolution in proteomic analysis of human serum samples. Proteomics 5:307-317, 2005.

12. Granger J, Siddiqui J, Copeland S, et al. Albumin depletion of human plasma also removes low abundance proteins including the cytokines. Proteomics 5:4713-4718, 2005.

13. Bjellqvist B, Ek K, Righetti PG, et al. Isoelectric focusing in immobilized $\mathrm{pH}$ gradients: principle, methodology and some applications. J Biochem Biophys Methods 6:317-339, 1982.

14. Corbett JM, Dunn MJ, Posch A, et al. Positional reproducibility of protein spots in two-dimensional polyacrylamide gel electrophoresis using immobilized $\mathrm{pH}$ gradient isoelectric focusing in the first dimension: an interlaboratory comparison. Electrophoresis 15:1205-1211, 1994.

15. Sanchez JC, Rouge V, Pisteur M, et al. Improved and simplified in-gel sample application using reswelling of dry immobilized $\mathrm{pH}$ gradients. Electrophoresis 18:324-327, 1997.

16. Li ZB, Flint PW, Boluyt MO. Evaluation of several two-dimensional gel electrophoresis techniques in cardiac proteomics. Electrophoresis 26:3572-3585, 2005.

17. Laemmli UK. Cleavage of structural proteins during the assembly of the head of bacteriophage T4. Nature 227:680-685, 1970.

18. Young D. Advantages of separation on "giant" two-dimensional gels for detection of physiologically relevant changes in the expression of protein gene-products. Clin Chem 30:2104-2106, 1984.

19. Switzer RC, Merril CR, Shifrin S. A highly sensitive silver stain for detecting proteins and peptides in polyacrylamide gels. Anal Biochem 98:231-237, 1979.

20. Neuhoff V, Arold N, Taube D, et al. Improved staining of proteins in polyacrylamide gels including isoelectric focusing gels with clear background at nanogram sensitivity using Coomassie Brilliant Blue G-250 and R-250. Electrophoresis 9:255-262, 1988.

21. Westermeier R, Marouga R. Protein detection methods in proteomics research. Biosci Rep 25:19-31, 2005.

22. Berggren K, Chernokalskaya E, Steinberg TH, et al. Backgroundfree, high sensitivity staining of proteins in one- and two-dimensional sodium dodecyl sulfate-polyacrylamide gels using a luminescent ruthenium complex. Electrophoresis 21:2509-2521, 2000.

23. Nishihara JC, Champion KM. Quantitative evaluation of proteins in one-and two-dimensional polyacrylamide gels using a fluorescent stain. Electrophoresis 23:2203-2215, 2002.

24. Luo L, Wirth PJ. Consecutive silver staining and autoradiography of ${ }^{35} \mathrm{~S}$ and ${ }^{32} \mathrm{P}$-labeled cellular proteins: applications for analysis of signal transducing pathways. Electrophoresis 14:127-136, 1993.

25. Unlu M, Morgan ME, Minden JS. Difference gel electrophoresis: a single gel method for detecting changes in protein extracts. Electrophoresis 18:2071-2077, 1997.

26. Lescuyer P, Allard L, Zimmermann-Ivol CG, et al. Identification of postmortem cerebrospinal fluid proteins as potential biomarkers of ischemia and neurodegeneration. Proteomics 4:2234-2241, 2004.

27. Schulenberg B, Goodman TN, Aggeler R, et al. Characterization of dynamic and steady-state protein phosphorylation using a fluorescent phosphoprotein gel stain and mass spectrometry. Electrophoresis 25:2526-2532, 2004.

28. Hart C, Schulenberg B, Steinberg TH, et al. Detection of glycoproteins in polyacrylamide gels and on electroblots using Pro-Q Emerald 488 dye, a fluorescent periodate Schiff-base stain. Electrophoresis 24:588-598, 2003.

29. Aksenov MY, Aksenova MV, Butterfield DA, et al. Protein oxidation in the brain in Alzheimer's disease. Neuroscience 103:373$383,2001$.

30. Castegna A, Thongboonkerd V, Klein JB, et al. Proteomic identification of nitrated proteins in Alzheimer's disease brain. $J \mathrm{Neu}$ rochem 85:1394-1401, 2003.

31. Aebersold RH, Leavitt J, Saavedra RA, et al. Internal amino-acid sequence-analysis of proteins separated by one-dimensional or two-dimensional gel-electrophoresis after in situ protease digestion on nitrocellulose. Proc Natl Acad Sci USA 84:6970-6974, 1987.

32. Montine TJ, Woltjer RL, Pan C, et al. Liquid chromatography with tandem mass spectrometry-based proteomic discovery in aging and Alzheimer's disease. NeuroRx 3:335-342, 2006 
33. Finehout EJ, Lee KH. An introduction to mass spectrometry applications in biological research. Biochem Mol Biol Educ 32:93100, 2004.

34. Finehout EJ, Lee KH. Comparison of automated in-gel digest methods for femtomole level samples. Electrophoresis 24:3508 3516, 2003.

35. Choe LH, Lee KH. Quantitative and qualitative measure of intralaboratory two-dimensional protein gel reproducibility and the effects of sample preparation, sample load, and image analysis. Electrophoresis 24:3500-3507, 2003.

36. Nishihara JC, Champion KM. Quantitative evaluation of proteins in one- and two-dimensional polyacrylamide gels using a fluorescent stain. Electrophoresis 23:2203-2215, 2002.

37. Molloy MP, Brzezinski EE, Hang J, et al. Overcoming technical variation and biological variation in quantitative proteomics. Proteomics 3:1912-1919, 2003.

38. Lilley KS, Razzaq A, Dupree P. Two-dimensional gel electrophoresis: recent advances in sample preparation, detection and quantitation. Curr Opin Chem Biol 6:46-50, 2001.

39. Garbis S, Lubec G, Fountoulakis M. Limitations of current proteomics technologies. J Chromatogr A 1077:1-18, 2005.

40. Beranova-Giorgianni S. Proteome analysis by two-dimensional gel electrophoresis and mass spectrometry: strengths and limitations. Trends Anal Chem 22:273-281, 2003.

41. Strohman R. Epigenesis: the missing beat in biotechnology. Biotechnology 12:156-164, 1994.

42. Finehout EJ, Franck Z, Lee KH. Towards two-dimensional electrophoresis mapping of the cerebrospinal fluid proteome from a single individual. Electrophoresis 25:2564-2575, 2001.

43. Sanchez JC, Appel RD, Golaz O, et al. Inside SWISS-2D PAGE database. Electrophoresis 16:1131-1151, 1995.

44. Tsuji T, Shimohama S. Analysis of the proteomic profiling of brain tissue in Alzheimer's disease. Dis Markers 17:247-257, 2001.

45. Fountoulakis M. Application of proteomics technologies in the investigation of the brain. Mass Spectrom Rev 23:231-258, 2004.

46. Vercauteren FGG, Bergeron JJM, Vandesande F, et al. Proteomic approaches in brain research and neuropharmacology. Eur J Pharmacol 500:385-398, 2004.

47. Gong CX, Liu F, Grundke-Iqbal I, et al. Post-translational modifications of tau protein in Alzheimer's disease. J Neural Transm 112:813-838, 2005.

48. Golde TE, Eckman CB, Younkin SC. Biochemical detection of A beta isoforms: implications for pathogenesis, diagnosis, and treatment of Alzheimer's disease. Biochim Biophys Acta 1502:172$187,2000$.

49. Kanninen K, Goldstein G, Auriola S, et al. Glycosylation changes in Alzheimer's disease as revealed by a proteomic approach. Neurosci Lett 367:235-240, 2004.

50. Butterfield DA, Abdul MH, Newman S, Reed T. Redox proteomics in age-related neurodegenerative disorders or models thereof. $\mathrm{Neu}$ roRx 3:343-356, 2006.

51. Markesbery WR. Oxidative stress hypothesis in Alzheimer's disease. Free Radic Biol Med 23:134-147, 1997.

52. Castegna A, Aksenov M, Aksenova M, et al. Proteomic identification of oxidatively modified proteins in Alzheimer's disease brain. Part I: creatine kinase BB, glutamine synthase, and ubiquitin carboxy-terminal hydrolase L-1. Free Radic Biol Med 33:562-571, 2002.

53. Cottrell BA, Galvan V, Banwait S, et al. A pilot proteomic study of amyloid precursor interactions in Alzheimer's disease. Ann Neurol 58:277-289, 2005.
54. Tilleman K, van den Haute C, Geerts H, et al. Proteomics analysis of the neurodegeneration in the brain of tau transgenic mice. Proteomics 2:656-665, 2002.

55. David DC, Hauptmann S, Scherping I, et al. Proteomic and functional analyses reveal a mitochondrial dysfunction in P301L tau transgenic mice. J Biol Chem 280:23802-23814, 2005.

56. Harrington MG, Merril CR, Asher DM, et al. Abnormal proteins in the cerebrospinal fluid of patients with Creutzfeldt-Jakob disease. $N$ Engl J Med 315:279-283, 1986.

57. Hsich G, Kenney K, Gibbs CJ, et al. The 14-3-3 brain protein in cerebrospinal fluid as a marker for transmissible spongiform encephalopathies. N Engl J Med 335:924-930, 1996.

58. Green AJE, Thompson EJ, Stewart GE, et al. Use of 14-3-3 and other brain-specific proteins in CSF in the diagnosis of variant Creutzfeldt-Jakob disease. J Neurol Neurosurg Psychiatry 70:744-748, 2001

59. van Everbroeck B, Boons J, Cras P. Cerebrospinal fluid biomarkers in Creutzfeldt-Jakob disease. Clin Neurol Neurosurg 107:355$360,2005$.

60. World Health Organization. Human transmissible spongiform encephalopathies. Wkly Epidemol Rec 73:361-365, 1998

61. Choe LH, Green A, Knight RSG, et al. Apolipoprotein E and other cerebrospinal fluid proteins differentiate antemortem variant Creutzfeldt-Jakob disease from antemortem sporadic CreutzfeldtJakob disease. Electrophoresis 23:2242-2246, 2002.

62. Davidsson P, Westman-Brinkmalm A, Nilsson CL, et al. Proteome analysis of cerebrospinal fluid proteins in Alzheimer patients. NeuroReport 13:611-615, 2002.

63. Davidsson P, Sjogren M, Andreasen N, et al. Studies of the pathophysiological mechanisms in frontotemporal dementia by proteome analysis of CSF proteins. Mol Brain Res 109:128-133, 2002.

64. Finehout EJ, Franck Z, Lee KH. Complement protein isoforms in $\mathrm{CSF}$ as possible biomarkers for neurodegenerative disease. Dis Markers 21:93-101, 2005.

65. Lescuyer P, Gandini A, Burkhard PR, et al. Prostaglandin D2 synthase and its post-translational modifications in neurological disorders. Electrophoresis 26:4563-4570, 2005.

66. Scott RB. Extraneuronal manifestations of Alzheimer's disease. J Am Geriatr Soc 41:268-276, 1993.

67. Choi J, Malakowsky CA, Talent JM, et al. Identification of oxidized plasma proteins in Alzheimer's disease. Biochem Biophys Res Commun 293:1566-1570, 2002.

68. Yu HL, Chertkow HM, Bergman H, et al. Aberrant profiles of native and oxidized glycoproteins in Alzheimer plasma. Proteomics 3:2240-2248, 2003.

69. Mattila KM, Frey H. Two-dimensional analysis of qualitative and quantitative changes in blood cell proteins in Alzheimer's disease: search for extraneuronal markers. Appl Theor Electrophor 4:189196, 1995.

70. Jabbour W, Pouplard-Barthelaix A, Houlgatte R, et al. Abnormal expression of actin in lymphocytes of Alzheimer's disease and Down's syndrome patients. J Neuroimmunol 38:199-208, 1992.

71. Rohlff C. Proteomics in molecular medicine: applications in central nervous systems disorders. Electrophoresis 21:1227-1234, 2000.

72. D'Ascenzo M, Relkin NR, Lee KH. Alzheimer's disease cerebrospinal fluid biomarker discovery: a proteomics approach. Curr Opin Mol Ther 7:557-564, 2005.

73. Listgarten J, Emili A. Practical proteomic biomarker discovery: taking a step back to leap forward. Drug Disc Today 10:1697$1702,2005$. 